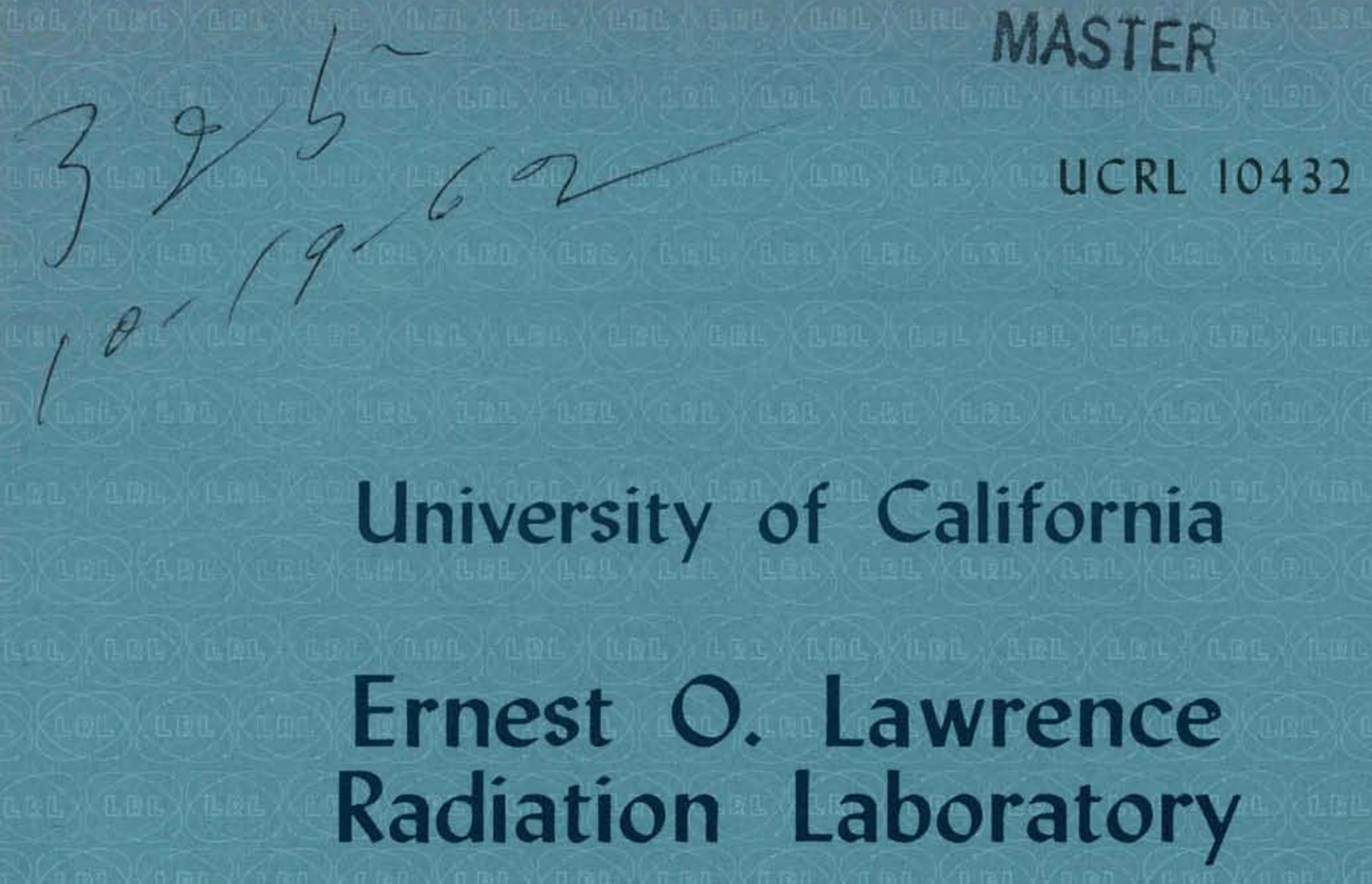

A SHELL MODEL THEORY OF THE R-MATRIX

Berkeley, California 


\section{DISCLAIMER}

This report was prepared as an account of work sponsored by an agency of the United States Government. Neither the United States Government nor any agency Thereof, nor any of their employees, makes any warranty, express or implied, or assumes any legal liability or responsibility for the accuracy, completeness, or usefulness of any information, apparatus, product, or process disclosed, or represents that its use would not infringe privately owned rights. Reference herein to any specific commercial product, process, or service by trade name, trademark, manufacturer, or otherwise does not necessarily constitute or imply its endorsement, recommendation, or favoring by the United States Government or any agency thereof. The views and opinions of authors expressed herein do not necessarily state or reflect those of the United States Government or any agency thereof. 


\section{DISCLAIMER}

Portions of this document may be illegible in electronic image products. Images are produced from the best available original document. 
UCRL - 10432

UC-4 Chemistry

TID -4500 (17.th Ed.)

\author{
UNIVERSITY OF CALIFORNIA \\ Lawrence Radiation.. Laboratory \\ Berkeley, California \\ Contract No. W $-7405-$ eng -48
}

A SHELL MODEL THEORY OF THE R-MATRIX

W. Tobocman

August 1962 

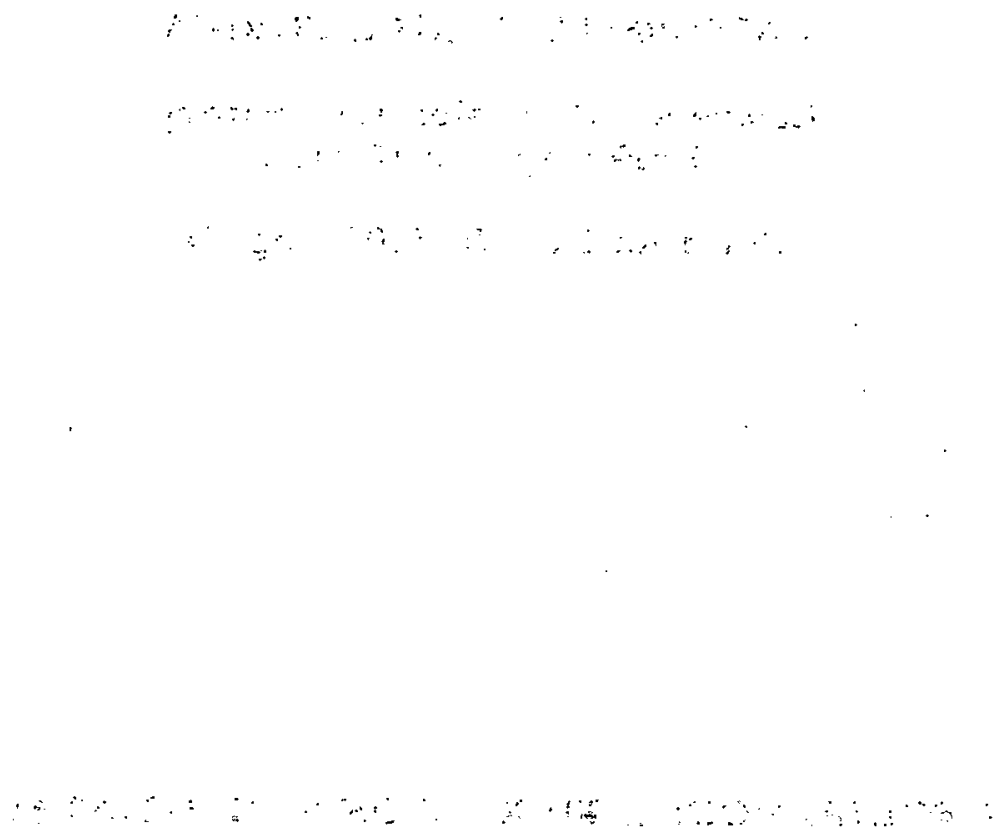

$\therefore \therefore$ : $\because \cdots$

Printed in USA. Price 50 cents. Available from the of fice of Technical Services U. S. Department of Commerce Washington 25 , D.C. 
A SHELL MODEL THEORY OF THE R-MATRIX

W. To.bocman

Lawrence Radiation Laboratory

University of California

Berkeley, California

\section{August 1962}

\section{ABSTRACT}

A method for dealing. with the nuclear many-body problem is suggested. The approach is a refinement of the shell model in which the asymptotic boundary conditions are used as constraints in a variational calculation of the R-matrix. Since it is the $\mathrm{R}$-matrix that is calculated, this method should provide a description of nuclear. reactions and decays as well as of nuclear bound states. 


\title{
A SHELL MODEL THEORY OF THE ·R-MATRIX
}

\author{
W. To boc man ${ }^{*}$ \\ Lawrence Radiation Laboratory \\ University of California \\ Berkeley, California
}

August 1962

\section{INTRODUCTION}

This paper will describe a procedure for making an approximate calculation of the $\mathrm{R}$-matrix. The suggested procedure is a modification of that used in shell model calculations. The $\mathrm{R}$-matrix is defined in terms of exact eigenfunctions of the Hamiltonian of a many-body system which are consistent with a specified set of boundary conditions. It is proposed to approximate. these exact eigenfunctions by finite linear combinations of known functions. The coefficients that appear in the se linear combinations are treated as variational parameters. They are determined by seeking the extrema of the expectation value of the Hamiltonian with the boundary conditions as constraints. By the method described here it should be possible to calculate the properties of nuclear. continuum states as well as the discreet states of nuclei. In this way. shell model techniques can be applied to nuclear reactions and nuclear decays as well as to the bound states of nuclei. This method should also make possible improvements in bound state calculations.

\section{THE R-MATRIX}

Suppose we have a system of $\mathrm{N}$. nucleons which has an energy low enough so that there are no open three-or-more-body channels. Let us denote the channel wave functions for this system by $x_{a}, x_{b}$ etc. Each channel wave function.. will consist of three factors.

$$
x_{a}=x_{a}^{A}\left(\hat{r}_{a}\right) x_{a}^{B} x_{a}^{C}
$$

$x_{a}^{B}$ is the wave function describing the internal degrees of freedom. of $n$ nucleons in a bound state while $x_{a}^{C}$ represents a bound state of the other

\footnotetext{
* Permanent address: Physics Dept. Case Institute of Technology, Cleveland 6, Ohio.
} 
$N-n$ nucleons. $x_{a}{ }^{A}$ is a function of the direction $\hat{r}_{a}$ of the vector $\stackrel{r}{r a}_{\text {giving }}$ the displacement of the center of mass of group $B$ from the center of mass of group C. Finally let us make $x_{a}$.an eigenfunction of the total angular momentum by appropriately coupling the orbital angular momentum represented by $x_{a}^{A}$ with the spins of $x_{a}^{B}$ and $x_{a}^{C}$, and let us make $x_{a}$ antisymmetric by applying an antisymmetrization operator to the product $x_{a}^{-{ }^{-}} x_{a}^{C}$.

For each channel wave function $x_{a}$ we define a channel radius $R_{a}$ such that when the magnitude $r_{a}$ of the relative displacement $r_{a}$ is greater than $\mathrm{R}_{\mathrm{a}}$, then the nuclear interaction between the two channel a clusters is negligible. Let $\mathrm{H}$ be the Hamiltonian for our system of $\mathrm{N}$ nucleons. We now define a set of "R-matrix wave:functions" $\Phi_{n^{\circ}}$ These functions will be exact eigenfunctions of the Hamiltonian

$$
H \Phi_{\mathrm{n}}=\mathrm{E}_{\mathrm{n}} \Phi_{\mathrm{n}}
$$

which satisfy the following boundary condition

$$
0=\left\{\frac{\mathrm{d}}{\mathrm{dr}} \mathrm{r}_{\mathrm{a}}\left\langle\dot{x}_{\mathrm{a}} \mid \Phi_{\mathrm{n}}\right\rangle-\left(\frac{\mathrm{L}_{\mathrm{a}}-1}{\mathrm{R}_{\mathrm{a}}}\right)\left\langle\mathrm{x}_{\mathrm{a}} \mid \Phi_{\mathrm{n}}\right\rangle\right\}_{\mathrm{r}_{\mathrm{a}}=\mathrm{R}_{\mathrm{a}}}
$$

for each channel. The logarithmic derivatives $L_{a}$ will be specified at a later stage of the discussion. The R-matrix can now be defined in the following way:

$$
R_{a b}=\sum_{n} \frac{\gamma_{a n} \gamma_{b n} A_{n}}{E-E_{n}}
$$

where

$$
\begin{aligned}
& \gamma_{a n}=\left\langle\chi_{a} \mid \Phi_{n}\right\rangle_{r_{a}=R_{a}} \sqrt{\frac{\hbar^{2} R_{a}}{2 M_{a}}} \\
& M_{a}=\text { the reduced mass in channel a } \\
& A_{n}^{-1}=\left\langle\Phi_{n}^{*} \mid \Phi_{n}\right\rangle_{R}=\int_{0}^{R} \mathrm{~d} \tau \Phi_{n} \Phi_{n}
\end{aligned}
$$

The normalization. integral $\dot{A}_{n}^{-1}$ is evaluated within a region whose boundaries are given by $r_{a} \leqslant R_{a}, r_{b} \leqslant R_{b}$, etc.

The $R$-matrix is related to the $\mathrm{S}$-matrix. The $\mathrm{S}$-matrix is descriptive of the asymptotic form of the solutions of our N-body Schroedinger equation.

$$
\mathrm{H} \Psi=\mathrm{E} \Psi
$$


In the asymptotic region

$$
\Psi \rightarrow \sum_{a} B_{a} \sum_{b} x_{b}\left(U_{b}^{(2)}\left(r_{b}\right) \delta_{b a}+U_{b}^{(1)}\left(r_{b}\right) s_{b a}\right)
$$

where the $B_{a}$ are constant coeficients and the $S_{b a}$ are the elements of the S-matrix.: The $U_{b}^{(2)}$ and $U_{b}^{(1)}$ are the unit-current radial wave functions of the incoming and outgoing types respectively. The relationship between the S-matrix and R-matrix can be shown to be of the form

$$
\delta_{b a}-R_{b a}\left(L_{a}-g_{a}^{(2)}\right)=\sum_{c} S_{c a} \frac{U_{c}^{(1)}\left(R_{c}\right)}{U_{a}^{(2)}\left(R_{a}\right)}\left\{R_{b c}\left(L_{c}-g_{c}(1)-\delta_{b c}\right\}\right.
$$

where

$$
g_{a}^{(i)}=\left[r_{a} \frac{d}{d r} \ln r_{a} U_{a}^{(i)}\left(r_{a}\right)\right]_{a}=R_{a}
$$

and

$$
\mathrm{L}_{\mathrm{a}}=\left[\mathrm{r}_{\mathrm{a}} \frac{\mathrm{d}}{\mathrm{dr}} \ln \mathrm{r}_{\mathrm{a}}\left\langle\mathrm{x}_{\mathrm{a}} \mid \Phi_{\mathrm{n}}\right\rangle\right]_{\mathrm{r}_{\mathrm{a}}=\mathrm{R}_{\mathrm{a}}}
$$

for ail $\Phi_{n^{*}}$

In the work of Wigner and his collaborators the logarithmic derivatives $\mathrm{L}_{\mathrm{a}}$ are chosen to be zero. Then the $\mathrm{R}$-matrix is real and equation (7) becomes

$$
\delta_{b a}+R_{b a} g_{a}^{(2)}=-\sum_{c} S_{c a} \frac{U_{c}^{(1)}\left(R_{c}\right)}{U_{a}^{(2)}\left(R_{a}\right)}\left\{R_{b c} g_{c}^{(1)}+\delta_{b c}\right\}
$$

In the work of Kapur. and Peierls the logarithmic derivatives $L_{a}$ are chosen to be equal to $\mathrm{g}_{\mathrm{a}}(\mathrm{i})$. Then the parameters in the $\mathrm{R}$-matrix become complex and dependent on $E$, but equation ( 7$)$ is considerably: simplified.

$$
S_{b a}=\frac{U_{a}^{(2)}\left(R_{a}\right)}{U_{b}^{(1)}\left(R_{b}\right)}\left\{R_{b a}\left(g_{a}^{(1)}-g_{a}^{(2)}\right)-\delta_{b a}\right\}
$$

The S-matrix has poles on the real axis and in the lower half of the complex plane (in energy). In the vicinity of one of these poles the S-matrix has the form

$$
S_{b a}=\frac{U_{a}^{(2)}\left(R_{a}\right)}{U_{b}^{(l)}\left(R_{b}\right)}\left\{\frac{i \sqrt{\Gamma_{a} \Gamma_{b}}}{E-E_{0}+i \frac{\Gamma}{2}}-\delta_{a b}\right\}
$$

where $\Gamma_{a}$ is the partial width for channel a and $\Gamma=\sum_{a} \Gamma_{a}$ is the total width. 
$\Gamma_{a}$ vanishes if channel, a is closed. Each such pole is associated with a. stable or metastable state of the $\mathrm{N}$-nucleon system, and the width $\Gamma$ is inversely proportional to the mean life of the state.

\section{CALCULATION OF THE R-MATRIX WAVE FUNCTIONS}

$\because$ Suppose $\Phi_{a^{\prime}} \Phi_{\beta^{\prime}} \cdots$ are a set of known, linearly independent, anti- : symmetrized, $\dot{N}$-nucleon wave functions. We propose to approximate: the $R$-matrix. wave functions $\Phi_{n}$ with linear combinations of the se known functions:

$$
\Phi_{\mathrm{n}}=\sum_{\mathrm{a}} \mathrm{B}_{\mathrm{a}}^{\mathrm{n}} \Phi_{\mathrm{a}}
$$

The coefficients $\mathrm{B}_{\mathrm{a}}^{\mathrm{n}}$ are regarded as variational parameters which are adjusted to yield extrema for the expectation value of the Hamiltonian." The boundary conditions given by equation (3) will be constraints on the variation. In addition, the normalization and orthogonalization condition

$$
\left\langle\Phi_{\mathrm{n}} \mid \Phi_{\mathrm{m}}\right\rangle_{\mathrm{R}}=\delta_{\mathrm{n}, \mathrm{m}}
$$

will al so constrain the variation.

To facilitate the discussion of the procedure for constructing the $\mathrm{R}$-matrix wave functions we introduce the following symbols:

$$
\begin{aligned}
& H_{a \beta}=\left\langle\Phi_{a}|H| \Phi_{\beta}\right\rangle{ }_{R} \\
& \mathrm{C}_{a \beta}=\left\langle\Phi_{a} \mid \Phi_{\beta}\right\rangle_{R} \\
& F_{a, a}=\left[\frac{d}{d r_{a}}\left\langle x_{a} \mid \Phi_{a}\right\rangle-\left(\frac{L_{a}-1}{R_{a}}\right)\left\langle x_{a} \mid \Phi_{a}\right\rangle\right] \underset{a}{r}=R_{a} \\
& M_{\alpha \beta}(E)=H_{\alpha \beta}-E G_{\alpha \beta} \\
& F_{a}^{n}=\left(\sum_{\beta} G_{a \beta} B_{\beta}^{n}\right)^{*}
\end{aligned}
$$

In terms of these quantities we have

$$
\left\langle\Psi_{\mathrm{n}}|\mathrm{H}| \Psi_{\mathrm{n}}\right\rangle=\sum \mathrm{B}_{\mathrm{a}}^{\mathrm{n}^{*}} \mathrm{H}_{\mathrm{a} \beta} \cdot \mathrm{B}_{\beta}
$$

for the expectation value of the Hamiltonian,

$$
\delta_{m n}=\sum B_{a}^{m}{ }^{*} G_{a \beta} B_{\beta}
$$


for the orthonormalization condition;

and

$$
\sum_{\beta} B_{\beta} F_{\beta b}=0
$$

for the boundary conditions.

To calculate the first or lowest energy $R$-matrix wave function we require

$$
\begin{aligned}
\delta\{ & \sum_{a \beta}\left(B_{a}^{1}{ }^{*} H_{a \beta} B_{\beta}^{1}-E_{1} B_{a}^{1}{ }^{*} G_{a \beta} B_{\beta}^{1}\right) \\
& \left.-\sum_{a a} B_{a}^{1 *} F_{a a}^{*} D_{a}-\sum_{\beta b} B_{\beta}^{1} F_{\beta b} D_{b}^{*}\right\}=0
\end{aligned}
$$

where $E_{l}$ and $D_{a}$ are Lagrange multipliers. The resulting equation for $\mathrm{B}_{\mathrm{a}}^{\mathrm{l}}$ is

$$
\sum_{\beta} M_{a \beta}\left(E_{l}\right) B_{\beta}^{1}=\sum_{a} F_{a a}^{*}: D_{a}:
$$

This is solved by inverting the matrix $M_{\text {a }}$

$$
B_{\dot{a}}^{1}=\sum_{\beta b} M_{a \beta}^{-1}\left(E_{1}\right) \cdot F_{\beta b}^{*} D_{b}
$$

The Lagrange multipliers $D_{a}$ are determined by the equations that result from substituting equation (19) into equation (16)

$$
\sum_{a \beta b} F_{a a} M_{a \beta}\left(E_{1}\right) F_{\beta b} * D_{b}=\sum N_{a b}\left(E_{l}\right) D_{b}=0
$$

The solution of this equation is found by diagonalizing the matrix $N\left(E_{1}\right)$ for the smallest value of $E_{1}$ that causes det $N\left(E_{1}\right)=0$. This procedure gives us $\mathrm{E}_{1}$ and the $\mathrm{D}_{a}{ }^{\prime}$ s to within a multiplicative constant. The normalization of the $D_{a}{ }^{i} s$ is fixed by the constraint

$$
\begin{aligned}
1 & =\left\langle\Phi_{1} \mid \Phi_{1}\right\rangle_{R}=\sum B_{a}^{1 *} G_{a \beta} B_{\beta}^{1} \\
& =\sum_{a \beta a} F_{\beta b} M_{a \beta}^{-1}\left(E_{1}\right)^{*} G_{a \gamma} M_{\gamma \delta}^{-1}\left(E_{1}\right) F_{\delta a}^{*} D_{b}^{*} D_{a} \\
& =\sum_{a b} J_{b a} D_{b}^{*} \cdot D_{a}
\end{aligned}
$$

Substituting these $D_{a}{ }^{9} s$ and $E_{1}$. back: into equations $(11)$ and (1.9) then gives $\Phi_{1}$ 。 
To calculate $\Phi_{2}$ we repeat the entire procedure outlined above except that the orthogonality of $\Phi_{2}$ and $\Phi_{1}$ provides an additional constraint. Thus the procedure for calculating $\Phi_{n+l}$ would yield the equations

$$
\begin{aligned}
& B_{a}^{n+1}=\sum_{\beta} M_{a \beta}^{-1}\left(E_{n+1}\right)\left\{\sum_{b} F_{\beta b}^{*} D_{b}+\sum_{\ell=1}^{n} F_{\beta}^{l *} D^{\ell}\right\} \\
& \sum_{a} B_{a}^{n+1} F_{a a}=0 \\
& \sum_{a} B_{a}^{n+1} F_{a}^{\ell}=0 \\
& \sum_{a \beta} B_{a}^{n+1} G_{a \beta} B_{\beta}^{n+1}=1
\end{aligned}
$$

The equations for $\Phi_{n+1}$ are seen to have the same structure as those for $\Phi_{1}$ except that there are $n$ additional Lagrange multipliers to be determined in the process of finding $\Phi_{n+1}$. There will, of course, be no more than $N$ linearly independent $\Phi_{n}^{\prime}$ 's that can be constructedin this: way, where $N$ is the number of $\Phi_{a}$ 's used. In terms of these $R$-matrix wave functions the

$$
\begin{aligned}
& R_{a b}=\sum_{n} \frac{\left\langle x_{a} \mid \Phi_{n}\right\rangle_{r_{a}=R_{a}}\left\langle x_{b} \mid \Phi_{n}\right\rangle_{r_{b}}=R_{b} \sqrt{\frac{\hbar^{4}}{4} \frac{R_{a} R_{b}}{M_{a} M_{b}}}}{\left(E-E_{n}\right) \cdot\left\langle\Phi_{n}^{*} \mid \Phi_{n}\right\rangle_{R}} \\
& =\sum_{n, a, \beta} \frac{\sqrt{\frac{\hbar^{4}}{4} \frac{R_{a} R_{b}}{M_{a} M_{b}}}\left\langle x_{a} \mid \Phi_{a}\right\rangle_{r_{a}}=R_{a}\left\langle x_{b} \mid \Phi_{\beta}\right\rangle_{r_{b}}=R_{b} B_{a}^{n} B_{\beta}^{n}}{\left(E-E_{n}\right\rangle \sum_{\gamma, \delta} B_{\gamma}^{n} B_{\delta}^{n}\left\langle\Phi_{\gamma}^{*} \mid \Phi_{\delta}\right\rangle_{R}}
\end{aligned}
$$




\section{IV.. CLUSTERING AND THE NUCLEAR SUREACE}

At this point we consider what choice would be best advised for the $N$. known $N$-nucleon. wave functions $\Phi_{a}$ to be used in constructing the $R$-matrix wave functions $\Phi_{n}$. : The first thing that comes to mind is the use of antisymmetrized-products of single particle orbitals just as is done in the shell model:- Such a representation has proved itself to be very successful in. describing the nuclear.interior. However; the shell model gives a very' inadequate picture of the nuclear surface. There are two reasons for this. First of all the harmonic oscillator wave functions customarily used for shell model orbitals fall off too rapidly in the asymptotic region. Secondly: and . most importantly clustering should occur at the nuclear surface where the nucleon density is reduced, and the se enhanced correlations in the surface :region cannot be adequately represented by a sum of a few products of single $\therefore$ particle orbitals:

To take a shell model wave function and force it to be consistent with realistic: boundary conditions by the procedure suggested in this paper. will probably not lead to an important improvement and will quite likely result in a worse result than the usual shell model calculation. This is just because the products of harmonic oscillator single particle orbitals are so poorly adpated to describe the nuclear surface. In order to implement the approach suggested by this paper it would seem necessary to include in the set of wave functions $\Phi_{a}$ types which contain clustering and also are enhanced in the surface region. These types. should serve to supplement and not displace the usual shellmodel types. At the very least, one should include clustering terms appropriate to each of the channels in which boundary; conditions are specified.

\section{DEFI NITION OF THE INSIDE REGION}

At various points in this paper symbols of the form $\langle\Phi|\theta| \Psi\rangle_{\mathrm{R}}$ have appeared. These are used to represent matrix elements of the type

$$
\langle\Phi|\sigma| \Psi\rangle_{\mathrm{R}}=\int_{0}^{\mathrm{R}} \mathrm{d} \tau \Phi^{*} \Theta_{\Psi}
$$

The symbol. $R$ is used to show that the integration is limited to the so-called inside region: $r_{a}<R_{a}, r_{b}<R_{b}, \cdots$. 
Now there is a certain ambiguity connected. with the definition: of the inside region; and this ambiguity is necessarily: present in any practical application of $\mathrm{R}$-matrix theory.

The two-body channel a is associated:with a particular clustering of: our $\mathrm{N}$-nucleon system into two groups $\mathrm{A}_{\mathrm{a}}$ and $\mathrm{B}_{\mathrm{a}}$.. The internal states of. these two groups of nucleons are represented by the wave:functions $x_{a}^{A}\left(\xi^{A}\right)$ and $x_{a}^{B}\left(\xi^{B}\right)$. $r_{a}$ denotes the displacement of the center of mass of. $A$ from that of $B$, while $\xi^{A}$ and $\xi^{B}$ represents the internal coordinates appropriate for clusters. $\mathrm{A}$ and $\mathrm{B}$. The meaning of cquation (16) w/ould be clear if we could write

$$
\int_{0}^{\mathrm{R}} \mathrm{d} \dot{\tau}=\int_{0}^{\mathrm{R}_{\mathrm{a}}} \mathrm{dr} \underset{\sim a}{ } \int \mathrm{d} \xi^{\mathrm{A}} \int \mathrm{d} \xi^{\mathrm{B}}
$$

for every channel a. But this cannot be simultaneously true for every channel $a, b, c, \cdots$ However, if the various channel radii. $R_{a}, R_{b}, \ldots$ are chosen to be sufficiently large and if the wave functions $\Phi_{a}, \Phi_{\beta}, \cdots$ are wisely selected, then the integrands of the integrals will always be negligible in the region where the several definitions of the inside region fail to correspond. 


\section{ACKNOW LEDGMENTS}

The author is particularly grateful to H. McManus for many critical discussions of the matters discussed in this paper. Thanks are also due to N. Glendenning and V. Gillet for helpful discussions.

This work was done under the auspices of the U. S. Atomic Energy Commission. 
This report was prepared as an account of Government sponsored work. Neither the United States, nor the Commission, nor any person acting on behalf of the Commission:

A. Makes any warranty or representation, expressed or implied, with respect to the accuracy, completeness, or usefulness of the information contained in this report, or that the use of any information, apparatus, method, or process disclosed in this report may not. infringe privately owned rights; ur

B. Assumes any liabilities with respect to the use of, or for damages resulting from the use of any information, apparatus, method, or process disclosed in this report.

As used in the above, "person acting on behalf of the Commission" includes any employee or contractor of the Commission, or employee of such contractor, to the extent that such employee or contractor of the Commission, or employee of such contractor prepares, disseminates, or provides access to, any information pursuant to his employment or contract with the Commission, or his employment with such contractor. 